\title{
Computational challenges in magnetic-confinement fusion physics
}

\author{
A. Fasoli ${ }^{\star}$, S. Brunner, W. A. Cooper, J. P. Graves, P. Ricci, O. Sauter and L. Villard
}

\begin{abstract}
Magnetic-fusion plasmas are complex self-organized systems with an extremely wide range of spatial and temporal scales, from the electron-orbit scales $\left(\sim 10^{-11} \mathrm{~s}, \sim 10^{-5} \mathrm{~m}\right)$ to the diffusion time of electrical current through the plasma $\left(\sim 10^{2} \mathrm{~s}\right)$ and the distance along the magnetic field between two solid surfaces in the region that determines the plasma-wall interactions $(\sim 100 \mathrm{~m})$. The description of the individual phenomena and of the nonlinear coupling between them involves a hierarchy of models, which, when applied to realistic configurations, require the most advanced numerical techniques and algorithms and the use of state-of-the-art high-performance computers. The common thread of such models resides in the fact that the plasma components are at the same time sources of electromagnetic fields, via the charge and current densities that they generate, and subject to the action of electromagnetic fields. This leads to a wide variety of plasma modes of oscillations that resonate with the particle or fluid motion and makes the plasma dynamics much richer than that of conventional, neutral fluids.
\end{abstract}

T he most straightforward way for describing plasmas would be the microscopic particle approach: solving the equations of motion for the many individual particles that form the plasma in externally imposed electromagnetic fields and in the fields that the particles themselves generate. However, this is computationally impossible to apply to realistic magnetic-fusion plasmas, which typically contain $10^{22}-10^{23}$ particles. (For a review of the basic concepts of magnetically confined fusion plasmas, see ref. 1.)

A statistical treatment leads to the kinetic model, based on the Vlasov equation (equation (1)), essentially Liouville's theorem applied to the specific plasma environment, with interactions that are dominated by electromagnetic fields and a separation between particle collisions - that is, interactions at microscopic scales-and long-range fields. The Vlasov equation describes the evolution of $f_{\mathrm{s}}(\mathbf{x}, \mathbf{v}, t)$, the single-particle phase-space distribution function of the species labelled ' $s$ ' (electrons or ions), under the effect of the longrange electric and magnetic fields $\mathbf{E}$ and $\mathbf{B}$, which evolve according to Maxwell's equations with plasma charge and current densities as sources:

$$
\frac{\partial f_{\mathrm{s}}}{\partial t}+\mathbf{v} \cdot \frac{\partial f_{\mathrm{s}}}{\partial \mathbf{x}}+\frac{q_{\mathrm{s}}}{m_{\mathrm{s}}}(\mathbf{E}+\mathbf{v} \times \mathbf{B}) \cdot \frac{\partial f_{\mathrm{s}}}{\partial \mathbf{v}}=0
$$

Here, $\mathbf{x}, \mathbf{v}, q_{\mathrm{s}}$ and $m_{\mathrm{s}}$ stand for the position, velocity, charge and the mass of a particle of species 's', respectively. In the Vlasov model, collisions are neglected, an approximation that is generally justified for fusion plasmas because small-scale effects based on Coulomb interactions become very weak at high temperatures. In fact, Coulomb collisions' cross-sections for the exchange of momentum and energy, and related quantities such as the electrical resistivity $\eta$, scale with $T^{-3 / 2}$. In ITER core plasmas (see ref. 1 ), for example, $T \approx$ $10 \mathrm{keV}\left(\approx 10^{8} \mathrm{~K}\right)$ and the electron-electron ('ee'), electron-ion ('ei') and ion-ion ('ii') collisional exchanges of momentum and energy (' $E$ ') occur over relatively long characteristic times: $\tau_{\mathrm{ee}}{ }^{E}=\tau_{\mathrm{ee}}{ }^{\mathrm{p}} \approx$ $\tau_{\mathrm{ei}}{ }^{\mathrm{p}} \sim 1 \mathrm{~ms} ; \tau_{\mathrm{ii}}{ }^{E}=\tau_{\mathrm{ii}}{ }^{\mathrm{P}} \sim 100 \mathrm{~ms} ; \tau_{\mathrm{ei}}{ }^{E} \sim 1 \mathrm{~s}$, resulting in $\eta_{\text {plasma }} \sim 0.1 \eta_{\mathrm{Cu}}$ (ref. 2)-justifying the collisionless Vlasov approach. For describing much denser and/or colder plasmas, such as in the edge region of a magnetically confined fusion plasma near the plasma-facing components of the vacuum chamber, or for describing phenomena occurring over longer timescales or situations when collisional effects are necessary to remove local phase-space singularities, a collisional operator with drag and diffusion terms accounting for the effect of multiple small-angle scatterings due to Coulomb interactions is added to the Vlasov equation, which then assumes the form of a Fokker-Planck equation. This form is also required for the description of not fully stripped impurities present in fusion devices and subject to inelastic collisional processes such as ionization, recombination and charge exchange.

By averaging the kinetic equations over the particle-velocity space, one can derive the equations that describe the evolution of fluid quantities. Multi-fluid models reflect the separate dynamics of the different species in the plasma, in particular ions and electrons.

By making further approximations, in part focusing on large spatial scales and low frequencies, one can construct a single-fluid, macroscopic model for the plasma and the magnetic field, known as the magnetohydrodynamic (MHD) model:

$$
\begin{gathered}
\frac{\partial \rho}{\partial t}+\nabla \cdot(\rho \mathbf{u})=0 \\
\rho \frac{\mathrm{d} \mathbf{u}}{\mathrm{d} t}=\mathbf{J} \times \mathbf{B}-\nabla p \\
\frac{\mathrm{d}}{\mathrm{d} t}\left(p \rho^{-\gamma}\right)=0 \\
\nabla \times \mathbf{E}=-\frac{\partial \mathbf{B}}{\partial t} \\
\mathbf{E}+\mathbf{u} \times \mathbf{B}= \begin{cases}0 & \text { Ideal MHD } \\
\eta \mathbf{J} & \text { Resistive MHD }\end{cases} \\
\nabla \times \mathbf{B}=\mu_{0} \mathbf{J} \\
\nabla \cdot \mathbf{B}=0
\end{gathered}
$$


a

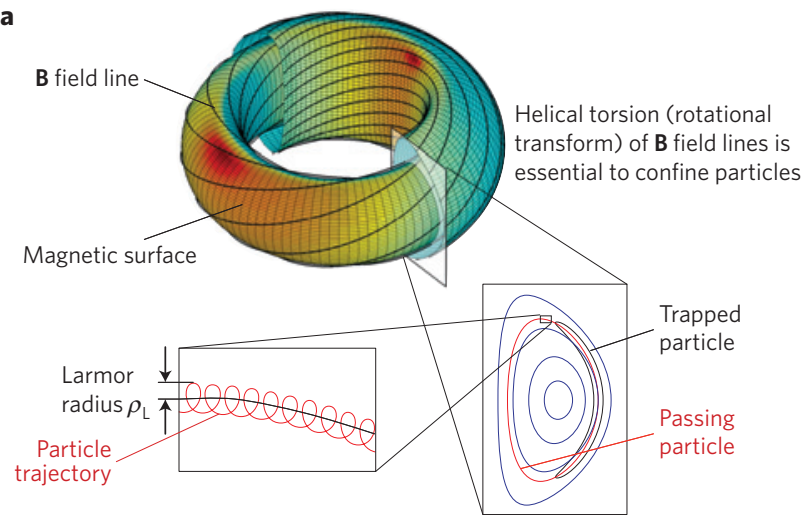

b

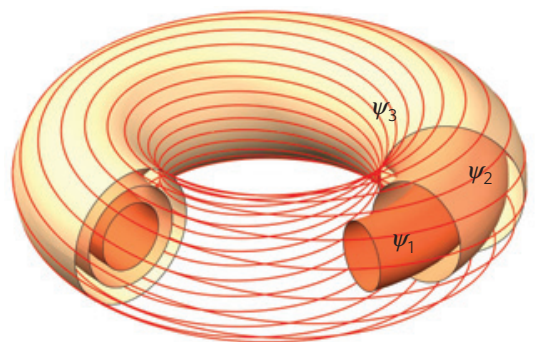

Figure 1 | Magnetic-field structure in a tokamak. a, Schematic view of the magnetic-field configuration and examples of particle trajectories. The image on the lower left shows a particle gyrating rapidly around a smooth 'guiding-centre' trajectory. The term 'trapped particles' refers to particles that, contrary to 'passing particles', are unable to complete a full poloidal turn, owing to the effect of the non-uniformity of the magnetic-field intensity, which acts as a magnetic mirror. b, Magnetic-field lines (red) and nested-flux surfaces, labelled by the value of the poloidal flux $\psi$, in a toroidal plasma equilibrium.

The MHD model describes the evolution of the electromagnetic field through a reduced set of Maxwell's equations valid for certain low-frequency phenomena, and the dynamics of the plasma current density $\mathbf{J}$, the average single-fluid velocity $\mathbf{u}$, the mass density $\rho$ and the plasma pressure $p$. The parameter $\gamma$ in the equation of state for $p$ and $\rho$ is referred to as the adiabatic exponent, in analogy with conventional fluids. For processes that can be considered as adiabatic, $\gamma$ corresponds to the ratio between specific heat at constant pressure and specific heat at constant volume, whereas in isothermal processes $\gamma=1$. We note that in plasma-transport simulations a full heat-balance equation is retained rather than this simple equation of state. The very low resistivity of the hot plasmas encountered in fusion research justifies in several cases the ideal MHD approximation $(\eta=0)$ (ref. 3), used for describing plasma equilibrium and stability and general phenomena that occur over timescales that are shorter than the resistive diffusion time for current through the plasma. It cannot describe, on the other hand, effects that occur over timescales comparable to the current diffusion time, such as those associated with changes in the topology of the magnetic field. The choice of the appropriate model for describing a particular plasma phenomenon constitutes an important aspect of the computational challenges in magneticfusion research.

This Review addresses a number of phenomena in magnetically confined plasmas that need to be understood both individually and in situations where they are coupled, including the equilibrium and stability of a plasma during build-up into a high-performance regime, heating scenarios, core and edge transport of thermal and non-thermal particles, the exhaust of plasma particles and power, and the interactions with the surrounding walls.

Although this is beyond the intended scope of this paper, we also recognize the crucial issue of modelling structural and functional materials for fusion ${ }^{4}$. Of particular importance are the effects associated with the interaction between the plasma and the first wall of a reactor, including erosion of the wall and the trapping of plasma fuel, which affects the tritium burn-up efficiency and increases to unacceptable levels the required tritium inventory. In addition, the high fluxes of $14.1 \mathrm{MeV}$ neutrons produced by the deuteriumtritium (DT) fusion reactions (a few $\mathrm{MW} \mathrm{m}^{-2}$ ) lead to structural modifications at the microscopic level (up to 15 displacements per atom per year in a fusion plant), which degrade the mechanical and thermal properties of the materials. (For a further overview on these issues-and of materials research in the context of nuclear fusion-see ref. 5.) As experimental results are very limited owing to the present unavailability of a neutron source reproducing the intensity and energy spectrum of a DT fusion reactor, numerical simulations play a key role. Computing radiation damage in fusion materials is also a challenging multi-scale problem, ranging from the atomic scales-that is, $10^{-9} \mathrm{~m}$ and $10^{-12} \mathrm{~s}$ - to the macroscopic size and lifespan of a fusion reactor. Results from simulations at the shorter scales provide essential information for setting parameters and properties of the reduced models employed for the larger scales. At the atomic scale, ab initio quantum-mechanical simulations are carried out to compute the electronic states of basic material configurations ${ }^{6}$. These computations provide interaction potentials, which are then used in classical molecular-mechanics simulations to study systems with a significantly larger number of atoms, in particular, displacement cascades induced by neutrons. Systems are studied at microscopic scales $\left(10^{-6} \mathrm{~m}\right.$ and $\left.10^{-6}-1 \mathrm{~s}\right)$ by means of kinetic Monte Carlo simulations, which are based on a statistical approach. Such simulations can address crystallinedefect nucleation, growth and migration, leading to microstructural changes of materials. Dynamic simulations are also carried out at these scales to study the interaction between dislocations and defects and to address the modification of the mechanical properties of the materials. Finally, materials properties at the macroscopic scale are simulated on the basis of diffusion-reaction models using finiteelement methods.

This Review intends to provide an overview of the computational challenges that the fusion community faces in view of reaching and controlling the burning-plasma regime. It is not meant to be a systematic review of the whole body of knowledge in this large field; we base our discussion on examples drawn from our experience, focusing on tokamaks and stellarators, with a focus on numerical predictions and applications for plasma control and optimization.

\section{Global equilibrium and stability of a tokamak}

At the most fundamental level, magnetic-fusion devices need to confine the charged plasma particles, that is, provide a magneticfield structure in which most particle orbits are contained. In addition to the gyrational motion of the plasma particles around the magnetic-field lines in a (complex) magnetic-confinement structure, different kinds of orbits feature disparate characteristic frequencies and sizes even for the same species, as illustrated in Fig. 1a for a tokamak magnetic-field configuration.

Once a scheme is devised that provides good single-particle confinement, a macroscopic equilibrium state must be found. As the characteristic times for the departure from equilibrium $(<100 \mu \mathrm{s})$ are much shorter than the time required for extracting fusion energy from a confined plasma, it is imperative that this equilibrium is macroscopically stable ${ }^{7}$. A variety of confinement systems have been analysed for equilibrium and stability ${ }^{8}$. We focus on the two most 

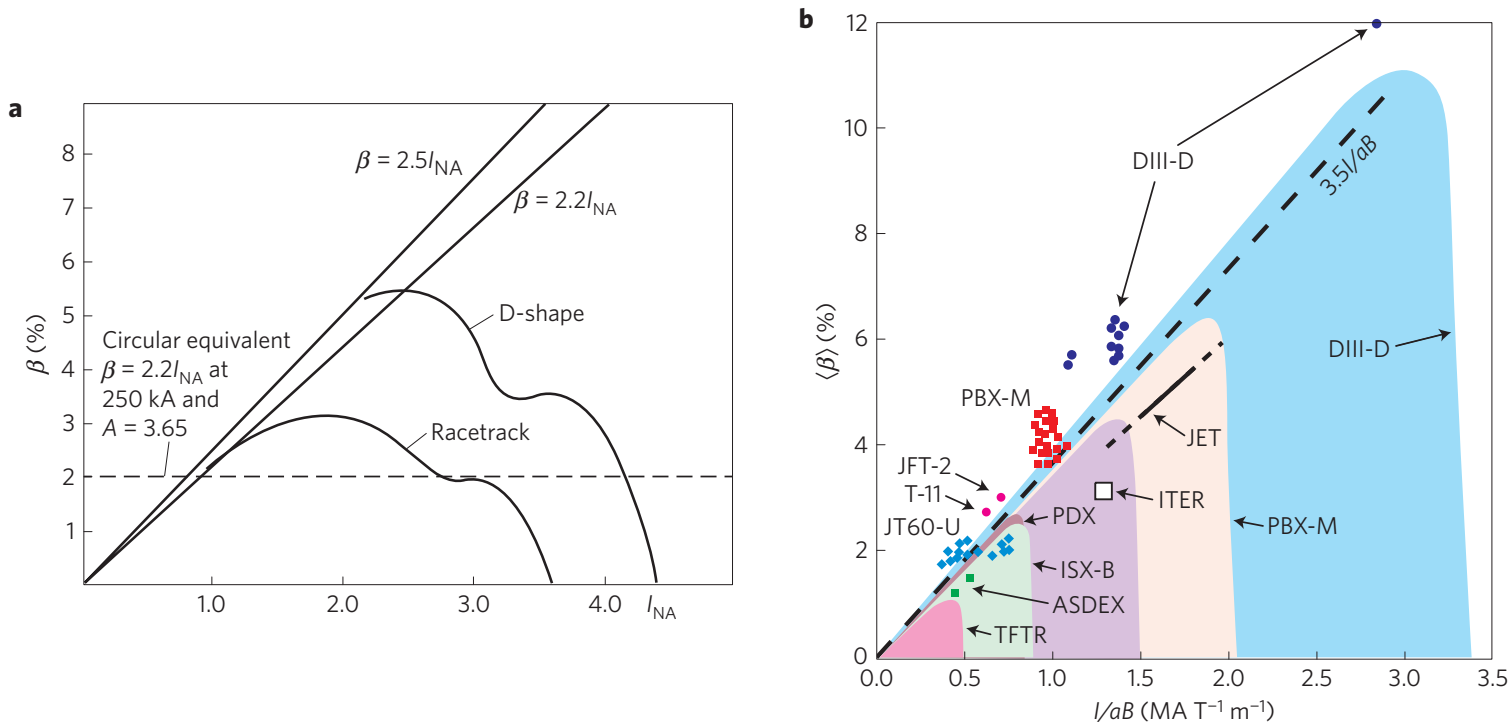

Figure 2 | Tokamak $\beta$-value limits. a, Prediction of the maximum stable $\beta$-value as a function of normalized plasma current $I_{\mathrm{NA}}=1 / \mathrm{AB}$, in $M A \mathrm{~T}^{-1} \mathrm{~m}^{-1}$, for the design of $\mathrm{TCV}^{18}$. A is the ratio of the major radius to the minor radius $a$ (the tokamak's aspect ratio). At high current, the $\beta$-limit deviates from the Troyon limit, indicated by the straight lines. The D-shaped tokamak plasmas have superior stability compared to the racetrack-shaped tokamak plasmas (originally considered because their shape would adapt to the rectangular confinement of the vacuum vessel, which would be beneficial for vertical stability). b. Stability diagram compiled from data for several tokamak devices, with the experimentally achieved value of volume-averaged $\beta$-values plotted as a function of the normalized plasma current. The shaded regions show the range of $\beta$ obtained in the respective experiments. Selected individual high- $\beta$ data are also shown for the respective experiments, as indicated by the arrows. The numerical factor in the scaling, 3.5, accounts for the effect of plasma non-circularity ${ }^{124}$. Reproduced from a, ref. 18, IOP; b, ref. 124, IOP.

promising schemes so far: the tokamak and (in the next section) the stellarator.

In a tokamak, a toroidal plasma is confined by twisted magneticfield lines, the curvature and gradient of which cause the particles to drift vertically in opposite directions for electrons and ions. The twist in the magnetic field, combined with a tokamak's axial symmetry, is essential in cancelling out these drifts on average: otherwise, charge separation would occur, creating a vertical electric field that would only partly be shielded out by the plasma dielectric behaviour and would cause all particles to drift radially outwards. Plasma confinement would be quickly lost. The toroidal component of the magnetic field ( $\sim 5 \mathrm{~T}$ in ITER) is produced by external coils, whereas the poloidal field component that provides the twist is generated by a current induced in the plasma (up to 15 MA in ITER) by transformer action (in a stellarator, this is achieved through additional magnetic coils).

Equilibrium and macroscopic stability calculations are typically performed within the MHD model, and have been among the first practical applications of numerical simulations in fusion plasmas. The characteristic time for the diffusion of magnetic-field lines across hot fusion plasmas is generally much longer than the time over which particles and energy are confined, thus the magnetic field can be considered frozen in with the plasma. Evaluating the dot product of the MHD static equilibrium condition obtained from force balance, $\nabla p=\mathbf{J} \times \mathbf{B}$ (see equation (2)), with $\mathbf{B}$ and $\mathbf{J}$ shows that the equilibrium magnetic-field lines lie on nested toroidal flux surfaces, which are isobaric and along which current flows. The centre of these nested surfaces, which are labelled by the value of the poloidal magnetic-flux function $\psi$ (see Fig. 1b), is the magnetic axis. Plasma quantities can be represented as a function of $\psi$ instead of the radial position. Assuming an axially symmetric configuration, the magnetic field can be expressed as $\mathbf{B}=F(\psi) \nabla \phi+\nabla \phi \times \nabla \psi$, where $\phi$ is the toroidal angle and the function $F(\psi)=R B_{\phi}(\psi)$, where $R$ is the radial position in the poloidal plane and $B_{\phi}$ the toroidal component of the magnetic field, relates mainly to the current in the external coils sustaining the toroidal magnetic field.
The nested-flux surfaces are enclosed within the last closed flux surface to the outside of which the so-called scrape-off layer (SOL) extends and where the magnetic geometry is characterized by open field lines, that is, lines that intersect the machine wall. The twist of the magnetic-field lines around the magnetic surface, referred to as the rotational transform, the inverse of which is called the safety factor, and its variation across the different magnetic surfaces, the magnetic shear, are key quantities for characterizing these configurations.

The MHD equilibrium condition and Maxwell's equations lead to the Grad-Shafranov equation ${ }^{9-11}$, a partial differential equation for $\psi$

$$
\begin{aligned}
{\left[R \frac{\partial}{\partial R} \frac{1}{R} \frac{\partial}{\partial R}+\frac{\partial^{2}}{\partial Z^{2}}\right] \psi } & =-\mu_{0} R j_{\phi}(R, Z) \\
& =-\mu_{0} R^{2} \frac{\mathrm{d} p(\psi)}{\mathrm{d} \psi}-F(\psi) \frac{\mathrm{d} F(\psi)}{\mathrm{d} \psi}
\end{aligned}
$$

Here $Z$ denotes the vertical position on the poloidal plane of the torus and $\mu_{0}$ is the magnetic permeability of free space. The function $F(\psi)$ and the pressure profile $p(\psi)$ yield the profiles of toroidal current density and of the safety factor. The peculiarity of this nonlinear equation is that $\mathrm{d} p / \mathrm{d} \psi$ and $F \mathrm{~d} F / \mathrm{d} \psi$ are free functions of $\psi$ that need to be provided to determine a given equilibrium solution-that is, the isobaric surfaces traced out over the poloidal cross-section, $\psi(R, Z)$. Initial guesses for the two functions are generally based on experimental knowledge of pressure and current density profiles.

We distinguish two classes of equilibrium computer codes: fixedand free-boundary. The free-boundary codes find the solution of equation (3) including the vacuum region, the wall and the magnetic-field coils. They are mainly used to reconstruct the equilibrium configuration given measurements of plasma density, temperature and currents in the coils, or to calculate the coil currents required to obtain a given plasma shape. Free-boundary 
codes account for the evolution of a plasma discharge and, having optimized their speed, are now routinely run in real time to assist plasma control systems ${ }^{12}$. For example, a free-boundary equilibrium reconstruction of the Tokamak à Configuration Variable (TCV) in Lausanne, Switzerland, is evaluated in a fraction of a millisecond ${ }^{13}$, which satisfies the real-time control needs. Information on the plasma equilibrium is usually the key part of complex real-time control systems, such as that of the Axially Symmetric Divertor Experiment-Upgrade (ASDEX Upgrade) tokamak ${ }^{14}$ in Garching, Germany, which includes real-time control of the microwave launcher for plasma heating and stabilization ${ }^{15}$. Fixed-boundary equilibrium codes, on the other hand, assume a given shape of the last closed flux surface and solve the Grad-Shafranov equation within this surface. They are used to provide very accurate equilibrium solutions, obtained by applying, for example, finiteelement methods with cubic basis functions ${ }^{16}$ for calculations of more complex features, such as stability, turbulence, heating and current drive.

The macroscopic plasma equilibrium can be become unstable owing to pressure gradients, curvature and gradient of the magnetic field, and current density, in particular when the pressure gradient and the magnetic curvature point in the same direction. Simulations of the maximum stable pressure associated with a given value of plasma current ${ }^{17}$, defining the Troyon $\beta$-limit, are generally used in the design of experimental devices. (The quantity $\beta$ is the ratio of the plasma pressure and the magnetic-field energy density-a measure for the confinement efficiency.) These calculations are based on the application of finite-element methods to the treatment of the plasma and magnetic energy as given by the MHD model, and determine the stability boundaries of a configuration by finding the absolute potential-energy minimum ${ }^{3}$. This was done, for example, when designing TCV, a device aimed at testing various plasma shapes and their effect on stability ${ }^{18}$. Plasmas with D-shaped cross-sections were found to be the optimum (Fig. 2a). It was found that it would be impossible to obtain stable plasmas with an elongation (the ratio of vertical to horizontal dimensions) greater than 3 , and that the $\beta$ limit increases with the plasma current ${ }^{17}$ only up to an elongation of about 2, above which it decreases. The record elongation obtained experimentally is 2.78 , an achievement occurring ten years after computational predictions ${ }^{19}$. The stability diagram constructed from measurements from many tokamak devices complies with simulation results (Fig. 2b). This kind of analysis has naturally also been performed for the design of ITER ${ }^{7}$, the equilibrium of which, for the $Q=10$ reference scenario ${ }^{1}$, is predicted to be characterized by a safety-factor profile going from 1 on the magnetic axis to about 3 near the edge, an elongation of $1.7-1.85$, and a $\beta$-value corresponding to about $60 \%$ of the Troyon limit.

Among the modes that can limit the achievable performance are global resistive modes, the description of which requires taking into account resistivity and two-fluid effects, or localized modes due to a combination of a flat safety-factor profile and large pressure gradients, referred to as infernal or long-lived modes. The saturated nonlinear structure of these modes can be described either by a nonlinear stability code that departs from an initially axially symmetric equilibrium, or, equivalently, by a three-dimensional (3D) code that finds the equilibrium condition by minimizing the total MHD energy (see below) ${ }^{20}$.

Steady-state reactor concepts usually require plasma operation above the ideal MHD stability limit. This is possible because of the stabilizing effect of the conducting device wall, as demonstrated experimentally ${ }^{21}$. However, because the wall has a finite resistivity, other types of modes can occur and limit the access to high $\beta$-values. The simulations of these effects are now so accurate that their results can be used to pacify instabilities during plasma operation using feedback control, although significant physics challenges related to kinetic effects remain.

\section{Global equilibrium and stability of 3D configurations}

The stellarator concept, devised in 1951, led to the first realization of a toroidal magnetic-confinement system. Stellarators require more complex optimization methods than tokamaks to realize configurations that have good confinement of thermal and suprathermal particles, good MHD stability properties, and minimal selfgenerated currents that could cause machine-sized magnetic islands (that is, magnetic-field structures that degrade confinement).

The development of optimized stellarators began around 1985, relying from the very beginning on numerical efforts. The confinement of trapped particles represents a challenge for conventional stellarators. In non-optimized stellarators, classical Coulomb collisions acting on the complex particle orbits result in so-called neoclassical effects, which, contrary to tokamak configurations, may dominate over turbulence in determining particle and energy cross-field transport. Calculations of neoclassical transport require properly accounting for the particle drift orbits and the Coulomb collisional operator. A major theoretical breakthrough (see ref. 22, and references therein) was the discovery that particles can be confined in the absence of exact axial symmetry for the magnetic field $\mathbf{B}$, as long as the magnetic field's strength $B$ has a coordinate of near-symmetry, similarly to the situation in tokamaks. This led to the design of configurations with a direction of near-symmetry, such as the quasi-helical symmetric stellarator ${ }^{23}$, or the quasi-axisymmetric stellarator ${ }^{24}$ (see, for example, ref. 25 for a survey of the approaches developed for transport mitigation in stellarators). The development of such magnetic configurations has been massively assisted by the introduction of magnetic coordinates ${ }^{26}$, for which the Jacobian of the non-orthogonal coordinate system depends on the magneticfield strength and is independent of the angular coordinates.

Another means of improving confinement in a configuration that does not have explicit symmetry properties is to limit particle drift motion across magnetic surfaces. This motivated the development of the quasi-isodynamic concept ${ }^{27}$, which features only a weak variation of $B$ in the poloidal coordinate, reducing the radial drift motion. This is in part the concept on which the design of the Wendelstein 7-X (W-7X) stellarator ${ }^{28}$ is based. W-7X has a major radius of $5.5 \mathrm{~m}$, a minor radius of $0.5 \mathrm{~m}$, a magnetic-field strength of $3 \mathrm{~T}$, and a heating power for the plasma of $14 \mathrm{MW}$; it is expected to confine plasmas up to volume average $\langle\beta\rangle \sim 5 \%$ during 30 -min pulses. First plasmas have recently been obtained in W-7X and the international fusion community is looking forward to experimentation on this device to prove the capability of the stellarator configuration to produce and confine reactor-grade plasmas, as tokamaks do, but with the potential advantage of having steady-state operation, no sudden losses of plasma confinement, and less violent instabilities, in particular at the plasma edge. In the quasi-isodynamic concept, the magnetic-field strength varies strongly in the toroidal direction, hence supra-thermal ions such as fusion-generated $\alpha$-particles $\left(\mathrm{He}^{2+}\right.$ nuclei) or those produced by auxiliary heating systems will still show finite radial motion. With this in mind, the W-7X stellarator has also been optimized to ensure that supra-thermal ions have closed orbits in the poloidal plane, as in tokamaks ${ }^{29}$.

It has recently been discovered, on the basis of numerical simulations, that tokamaks can also evolve into $3 \mathrm{D}$ configurations. Persistent core-localized structures have been observed in tokamaks, which are the manifestations of a saturated ideal internal 3D mode $\mathrm{D}^{30,31}$.

\section{Heating and current drive by waves}

Having confined individual particles and constructed a macroscopically stable equilibrium, one must consider how to heat the plasma to thermonuclear temperatures $(T \sim 10-30 \mathrm{keV})$. The vanishing plasma resistivity at high temperatures prevents 


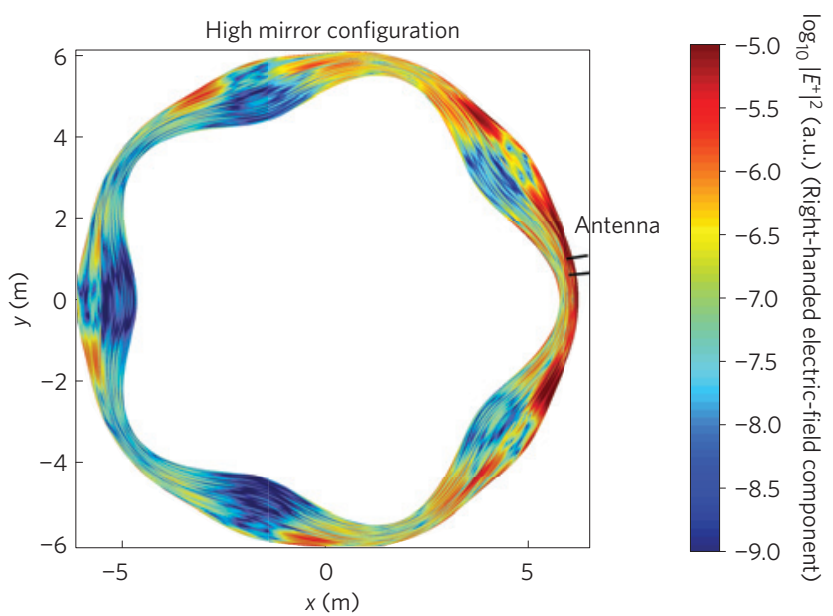

Figure $\mathbf{3}$ | Simulating ion cyclotron-resonance heating. Shown is the structure of the wave launched with a frequency of $33.8 \mathrm{MHz}$ by an antenna (the location of which is indicated) to heat the plasma in the W-7X stellarator. The square of the modulus of the left-handed electric-field component $E^{+}$at the equatorial mid-plane of the device is plotted. The magnetic-field strength is $2.5 \mathrm{~T}$; the wave resonates with hydrogen, a minority species (concentration 1\%) in a helium plasma with central density $8 \times 10^{19} \mathrm{~m}^{-3}$ and temperature $3 \mathrm{keV}$. Reproduced from ref. 39, American Institute of Physics.

an effective use of resistive (ohmic) heating, which is usually insufficient to counteract the radiation and conduction/convection losses for $T>1 \mathrm{keV}$. Additional heating schemes have therefore been devised, based on the injection of highly energetic neutral particles (neutral-beam injection, NBI), or waves ${ }^{2,32}$.

The variety of characteristic temporal and length scales in magnetic-fusion plasmas is paralleled by the richness of the spectrum of electromagnetic waves that can propagate through them $^{33}$. Different plasma models account for different modes of oscillation. Even in the single-fluid MHD model, a range of propagating waves exist, like those that are akin to ordinary sound waves, combinations of sound and magnetic waves, and shear Alfvén waves, which result from a combination of the tension of magnetic-field lines and plasma inertia.

To describe modes specifically associated with the plasma ion or electron dynamics, one needs to use a two-fluid model. A variety of modes are found for the different propagation directions with respect to the ambient magnetic field. Some plasma waves (called electrostatic waves) are characterized by a low phase velocity (much lower than the electron thermal speed, which in turn is much lower that the speed of light) and their oscillating magnetic component can be neglected.

Waves play a crucial role in plasmas, because many modes naturally become unstable because of the large free-energy sources that are present in confined plasmas, such as current density and pressure gradients (in ITER, the current density at the core is about $10 \mathrm{MA} \mathrm{m}^{-2}$ and the current density at the core is about $100 \mathrm{MPa} \mathrm{m}^{-1}$ ), and develop nonlinearly into small-scale turbulent fluctuations around the equilibrium states. Turbulence is responsible for most of the transport of heat and particles across the magnetic field of magnetically confined fusion plasmas. To optimize the plasma fusion performance it is therefore important to understand and (at least partly) control these nonlinear features. Waves that develop into turbulence have frequencies that lie well below the ion cyclotron frequency and often exhibit electrostatic character. A general example is provided by the broad category of drift waves, which become unstable owing to density and temperature gradients across the magnetic field.
To efficiently heat or drive current in the plasma, one can use waves that resonate with the electron or ion motion. Fluid resonances correspond to a wave's index of refraction going to infinity and lead to absorption of the wave's energy. In the context of the two-fluid model, a scheme needs to be devised in which an injected wave accesses a resonance before meeting a cutoff point, at which the index of refraction vanishes and the wave is reflected.

The concept of electron and ion cyclotron-resonance heating (ECRH and ICRH, respectively) is based on transferring power (tens of megawatts in ITER and future reactors) from external antennas to the plasma by launching waves, the frequency of which matches the cyclotron frequency (or its harmonics) of electrons or of a particular ion species at a specific location inside the plasma.

In the case of ICRH, the cyclotron frequency of hydrogen or helium-3 ions in magnetic fields of the order 3-6 T, such as in ITER, is in the range $45-90 \mathrm{MHz}$, which corresponds to wavelengths that are comparable to the machine size. Global wave computations are therefore needed. A mode that can propagate in the plasma at this frequency is the fast magneto-sonic wave, a branch of the two-fluid dispersion relation that is characterized by an oscillating electric field that is perpendicular to the ambient magnetic field. As the fast magneto-sonic wave is evanescent below a certain density, and the antenna cannot be inserted in the plasma, the wave needs to tunnel through an edge cutoff region to reach the plasma core.

While travelling along the magnetic field, the plasma ions gyrate anticlockwise and can resonate with the electric field with anticlockwise, left-handed, circular polarization, $E^{+}$. Unfortunately, plasma collective effects screen the left-handed polarized wave at the location where the majority of ions would be in resonance with it. Heating of the majority plasma ions could still be possible through a mode-conversion process-that is, a linear transfer of energy between modes where the relevant dispersion relations coincide. However, a more efficient alternative is to select a minority species with low concentration that has a cyclotron frequency different from that of the main ion species, thus resonating with the wave at a location where the left-handed component of the wave is only weakly screened by the plasma. The energy deposited to the minority ions is then transferred to electrons and to the main species ions by collisional processes.

A novel ICRH heating approach, at present investigated in modelling and in experiments, is the three-ion scheme ${ }^{34}$, for which the cyclotron resonance of small concentration minority species is situated between those of two separate majority species. Theory shows that in this scenario it is possible to maximize the left-handed component $E^{+}$of the wave and thus optimize the power absorption. This has very favourable implications for DT reactor plasmas to which very small concentrations of ${ }^{7} \mathrm{Li}$ ions are added.

To model ICRH, codes need to calculate the wave field in a plasma comprised of multiple ion species and electrons, and compute the transfer of energy to the ion population. The heated ions affect in turn the wave propagation and damping, and the force balance of the equilibrium magnetic field. Therefore, the computational procedure for ICRH is based on iterations between the calculation of the wave, of the particle phase-space distribution, and of the plasma equilibrium. At present, numerous $\operatorname{codes}^{35,36}$ are capable of iterating between the first two, mostly considering an isotropic background equilibrium and ignoring finite orbit effects, although significant efforts to circumvent these limitations have recently been developed ${ }^{37}$.

The most difficult challenges that remain in simulating ICRH in tokamaks and stellarators relate to the coupling between the antenna and the edge plasma, which involves strongly nonlinear effects associated with the large radiofrequency (RF) voltages (tens of kilovolts) present at the antenna surface, and the turbulent nature of the edge-plasma region ${ }^{38}$. This coupling strongly influences not only the effectiveness of the heating, but also the integrity of the 


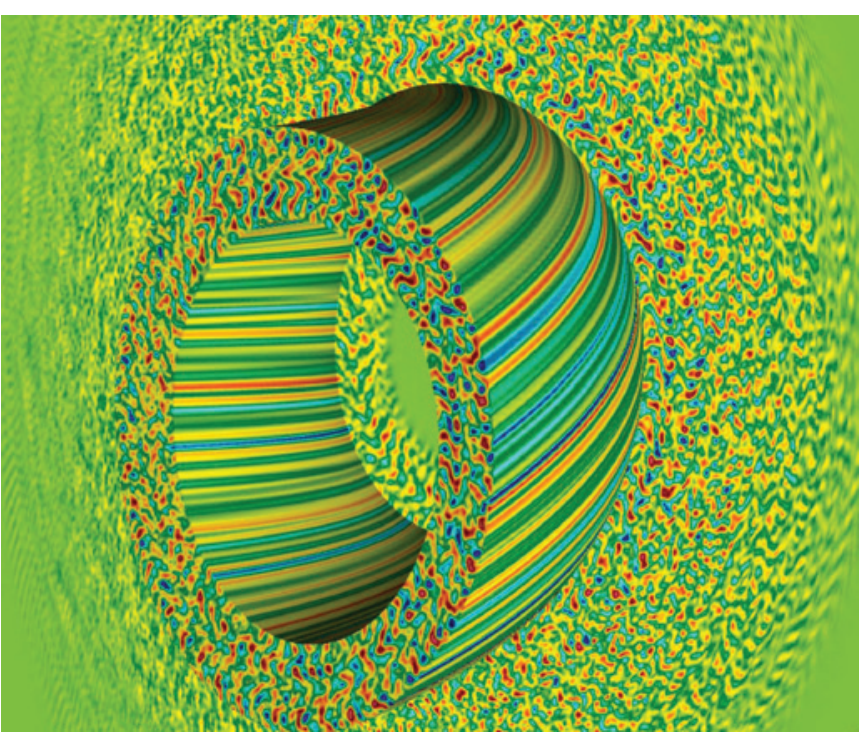

Figure 4 | Gyrokinetic simulation of ITER plasmas. Snapshot of the perturbed electron density from a simulation of ion-scale plasma turbulence in the ITER tokamak core ${ }^{125}$. Turbulence was simulated using the ORB5 global gyrokinetic code ${ }^{54}$. A cut-away view of a set of O-shaped magnetic surfaces visualizes the 3D turbulence structures. The colour scale for the ratio of the density perturbation to the background value goes from blue (minimum value, $-1 \%$ relative deviation) to red (maximum value, $+1 \%$ relative deviation).

antenna, which is subject to electrical arcs and ion bombardment due to the rectification of the RF voltage in the plasma sheath close to the antenna, and can strongly modify the properties of the edge plasma.

In a stellarator such as $\mathrm{W}-7 \mathrm{X}$, the localization of the antenna produces two important additional numerical challenges. First, the toroidal periodicity is broken: the pressure associated with the heated ions and the thermal ions is not periodic in the toroidal direction. In particular, in nearly quasi-isodynamic stellarators (such as W-7X), certain classes of particles are not free to move toroidally through the plasma and, as a result, the pressure associated with the heated ions can be enhanced more in the portion of the stellarator configuration that contains the antenna. Second, whereas massive parallel processing is required for calculating the fast-particle trajectories in an ICRH code (with almost linear scaling of speed-up with respect to the number of processors, which runs into the thousands), many platforms do not have sufficient shared memory on each node to compute the wave field. The number of modes required to represent the wave fields of realistic antenna models becomes very large. State-of-the-art simulations ${ }^{39}$ do indicate that the heat absorption concentrates in the part of the torus adjacent to the antenna, as highlighted by the distribution of the electric field $E^{+}$in Fig. 3. Similarly, modelling ICRH in tokamak plasmas that are strongly $3 \mathrm{D}$ in the core is very challenging owing to the large spectrum of modes required to represent the equilibrium ${ }^{40}$.

In the ECRH case, electromagnetic waves are launched at much higher frequencies, as the electron-cyclotron frequency in devices with a magnetic field of $3-6 \mathrm{~T}$ is in the $90-180 \mathrm{GHz}$ range. Energy is transferred from the wave to the perpendicular motion of the electrons. If the waves are launched with a finite angle along the magnetic field, the wave-particle interaction, which can be modelled using the Fokker-Planck equation, can lead to an asymmetric distribution function in the parallel directionthat is, to an electrical current. This method, referred to as electron-cyclotron current drive (ECCD), has been demonstrated experimentally in a number of devices, and intensive code benchmarking has been performed ${ }^{41}$. In the TCV tokamak ${ }^{42}$, the whole plasma current has been sustained by ECCD up to recharging of the ohmic transformer. ECRH and ECCD are at present considered as the main methods for plasma heating and local current drive on ITER and future demonstration (DEMO) reactors, in addition to NBI.

Many codes with various degrees of complexity have been developed to describe the interaction between the launched electron-cyclotron wave and the plasma ${ }^{41}$. In contrast to the ICRH case, the electron-cyclotron waves propagate both in vacuum and in the plasma, and the wave path up to the resonant region in the plasma core is relatively straightforward to calculate. The coupling to the plasma is generally properly accounted for by the simulations. The computational challenges now mainly reside in the calculation of the details of the deposition profiles of the electron-cyclotron power, in particular considering the very large local power densities ( $\sim 1-10 \mathrm{MW} \mathrm{m}^{-3}$ in ITER), and the related nonlinear effects. The ECCD can modify the current density profile very locally. It is therefore ideal for stabilizing plasma modes and for controlling the current profile, but the degree of locality is difficult to assess. For example, it has been shown that turbulence, in particular density fluctuations, can scatter the electron-cyclotron waves and broaden the effective deposition width ${ }^{27}$. This mechanism is particularly significant when the beam travels over long distances in the plasma, as will be the case in ITER. In addition, the effect of diffusion due to micro-turbulence in configuration and velocity space is a challenge, requiring calculations based on the Fokker-Planck equation. These effects can impact the capability of ECCD to control instabilities by locally acting on the current profile ${ }^{43}$.

\section{Core-plasma transport}

To describe the exchange of energy and momentum between the particles and the waves that are injected from antennas and those that are unstable and develop into turbulence, leading to anomalous (that is, non-collisional) transport, more refined models than those based on fluid-dynamics equations are needed. Kinetic models with the appropriate level of detail are derived from combining Maxwell's equations for the electromagnetic fields with the Vlasov or Fokker-Planck equation. Depending on the application, an averaging process over the fast-particle gyrational motion can be justified, to reduce the phase-space dimensionality and to remove the fast cyclotron timescale. Several codes have been developed for implementing such gyrokinetic models, most of which with the purpose of describing the turbulence and the plasma transport in the core region ${ }^{44}$.

The presence of a background magnetic field implies an extreme anisotropy of the plasma-transport properties: transport is several orders of magnitude faster in the parallel direction than across the magnetic field. In the plasma core, the magneticfield lines lie on closed magnetic surfaces (see Fig. 1), and losses can occur only across them. Experimentally measured heat and particle fluxes from the core to the periphery of magnetically confined fusion plasmas are one to two orders of magnitude larger than predicted by so-called neoclassical transport theory, which takes into account only collisional processes affecting the particle trajectories. In a tokamak device such as the Joint European Torus (JET) in Culham, UK, the ion heat diffusivity is of the order of $1 \mathrm{~m}^{2} \mathrm{~s}^{-1}$, whereas the neoclassical value would be smaller than $0.1 \mathrm{~m}^{2} \mathrm{~s}^{-1}$. Furthermore, the observed scaling of transport coefficients with some key physical parameters is also incompatible with collisional theory. This anomalous transport of particles, heat and momentum (related to plasma rotation) results from a turbulent state called micro-turbulence, which is characterized by density and temperature fluctuation levels of just a few percent of the background values ${ }^{44}$. Micro-turbulence is driven by low-frequency modes that are destabilized by density 
or temperature gradients and inhomogeneities of the magnetic field $^{45}$. The associated fluctuations are strongly elongated along the magnetic field-highly anisotropic-with long (machine-size) parallel wavelengths and short (of the order of the ion or electron Larmor radius) perpendicular wavelengths (Fig. 4). This anisotropy reflects the fact that particles are highly mobile along the magnetic field but undergo slow perpendicular drifts.

Accounting for the different dynamics of the various plasma species is essential for describing micro-instabilities. As the plasma core is characterized by low collisionality, intrinsically kinetic phenomena, such as non-collisional Landau damping or effects related to the finite size of the particle Larmor radii, play a key role, and call for a multi-species kinetic model. The difficulty of modelling plasma micro-turbulence in a kinetic framework is further compounded by the complex topology of the equilibrium magnetic fields, and by the diverse nature of the various types of micro-instabilities that may simultaneously drive the turbulence at the ion- and electrondynamics temporal and length scales. The most general kinetic representation would involve solving the Vlasov or Fokker-Planck equation to determine $f_{s}(\mathbf{x}, \mathbf{v}, t)$. However, a more practical description, referred to as gyrokinetic theory, can be derived by invoking the above-mentioned temporal and spatial scaling properties of microturbulence ${ }^{46}$. The derivation of the gyrokinetic equations involves averaging the particle trajectories over the fast cyclotron rotation, which leads to an evolution equation for the distribution of the particles' centres of gyro-rotation, called guiding centres, while still retaining information on finite Larmor radius effects when estimating associated charge densities and currents. The main advantages of a gyrokinetic description are that the effective phase space is reduced from six to five dimensions, and that fast cyclotron timescales are eliminated and need not be resolved. Gyrokinetic theory began with the pioneering local approach of Frieman and $\mathrm{Chen}^{47}$ and has been extended in various ways over the years ${ }^{48}$. Non-local versions of gyrokinetic theory have been derived using Hamiltonian- and Lagrangian-field-theory methods ${ }^{46,49}$. For recent introductions to gyrokinetic theories, see refs 44,50 .

The impressive development of high-performance computing resources in the past decades has been essential for the progressive introduction of enhanced realism into micro-turbulence simulation models. In the mid-1980s to mid-1990s, owing to the limited numerical resources, most computations addressed only the linear evolution. First nonlinear simulations were attempted for the most basic scenario-that is, turbulence driven by ion temperature gradient (ITG) modes, which are essentially sound waves destabilized by ion temperature gradients. For these low-frequency electrostatic fluctuations, ion dynamics must be described gyrokinetically, but electrons may be assumed to respond adiabatically, so that their density follows a linearized Boltzmann response: $n_{\mathrm{e}}(\mathrm{x}, t)=n_{0 \mathrm{e}} \exp \left[e\left(\phi-\phi_{0}\right) / T_{0 \mathrm{e}}\right] \approx n_{0 \mathrm{e}}\left[1+e\left(\phi-\phi_{0}\right) / T_{\mathrm{e}}\right]$, with $n_{0 \mathrm{e}}(\psi)$ and $T_{0 \mathrm{e}}(\psi)$ the background-density and electrontemperature profiles, respectively, $e$ the elementary charge, $\phi(\mathbf{x}, t)$ the fluctuation electrostatic potential and $\phi_{0}(\psi, t)$ the flux-surface average of $\phi(\mathbf{x}, t)$.

Historically, the first approach adopted to solve the gyrokinetic equations was based on the Lagrangian particle-in-cell (PIC) representation $^{51}$, in which a set of numerical particles are pseudo-randomly distributed in phase space and are followed along their respective orbits, whereas the self-generated electromagnetic fields are obtained from grid-based solvers. A low-noise PIC method, the so-called delta-f approach, was specifically developed and applied for this purpose. These pioneering computations enabled important physical insights to be obtained into some key turbulent mechanisms, in particular on how turbulence can generate and maintain poloidal, or zonal, flows in the plasma that rotate in opposite directions in different radial zones. These flows, in turn, shear the turbulent eddies, resulting in self-regulation of the turbulence and a strong reduction of the associated transport ${ }^{52,53}$. Under certain conditions, such shear flows can generate radial regions of significantly reduced turbulent transport, referred to as transport barriers.

In the late 1990s, the available HPC resources increased enormously thanks to the development of parallel computing. Not only did this development benefit PIC-based approaches ${ }^{54}$, but it also encouraged the implementation of grid-based approaches for carrying out gyrokinetic simulations ${ }^{55-57}$. The significant advantage of such an Eulerian over a Lagrangian particle-type representation is the absence of numerical sampling noise. An intermediate approach between particle- and grid-based calculations, the semi-Lagrangian approach, has also been developed ${ }^{58}$. Lagrangian, Eulerian and semi-Lagrangian approaches each have their own advantages and disadvantages ${ }^{44}$. Given the importance and difficulty of the turbulence problem, it is essential to pursue the development of all these different schemes.

Gyrokinetic computations are costly, therefore first simulations were carried out in the so-called flux-tube geometry, which consists of a small, elongated sub-volume of the core plasma, following a particular magnetic-field line. The local flux-tube model assumes a scale separation between the size of turbulent eddies (a few Larmor radii long) and the much larger characteristic lengths of variation of the background plasma (of the order of the minor radius of the toroidal plasma $)^{59}$. Nowadays, gyrokinetic codes no longer need to rely on certain hypotheses of scale separation and can address global effects, which are particularly important in smaller devices and, in general, in regions of strong and localized gradients such as the region near the plasma-edge and internal transport barriers.

Eulerian codes are well suited to address the effects related to kinetic electron dynamics on micro-turbulence, as these are strongly subject to numerical noise, although one should mention recent work on improved algorithms for PIC computations including electromagnetic effects ${ }^{60,61}$. Accounting for kinetic electrons, instead of adopting a simple adiabatic-response model, results in a much wider range of possible waves and instability mechanisms. The trapped-electron mode (TEM), for example, is an electrostatic wave that becomes unstable through a resonant wave-particle process, involving the toroidal precessional drift of trapped electrons in the presence of electron temperature or density gradients. TEMs are mid-range between ion and electron scales. Another important electrostatic micro-instability driven by electron dynamics is the electron-temperature gradient (ETG) mode, which can be seen as the electron analogue of the ITG mode. According to our current understanding, ITGs, TEMs and ETGs all play a role in turbulent transport processes, although their relative importance is still far from being fully understood.

The interaction between ion- and electron-scale driven fluctuations is therefore an important open question. Recent research shows ${ }^{62}$ that electron-scale turbulence, characterized by radially elongated eddies called streamers, can sometimes ${ }^{63}$ be suppressed by ion-scale turbulent eddies, and thus the transport of both ions and electrons is dominated by ion-scale turbulence. On the other hand, when ion-scale modes are stabilized by the plasma pressure, electron-scale turbulence increases and damps the ion-scale zonal flows, leading in turn to an enhancement of ion-scale turbulent transport. Owing to the heavy computational requirements, such multi-scale simulations have so far been carried out only in local flux-tube codes, even though global effects may play an essential role in determining turbulent transport levels ${ }^{64,65}$.

Another challenging field of investigation is related to plasma rotation: plasmas are observed to spin up even in the absence of applied external torque. Such intrinsic rotation is observed to reach toroidal velocities of the order of $50 \mathrm{~km} \mathrm{~s}^{-1}$ in several presentday tokamaks ${ }^{66}$. Plasma rotation is an important element in the self-organization of the plasma, owing to its shearing effect on 


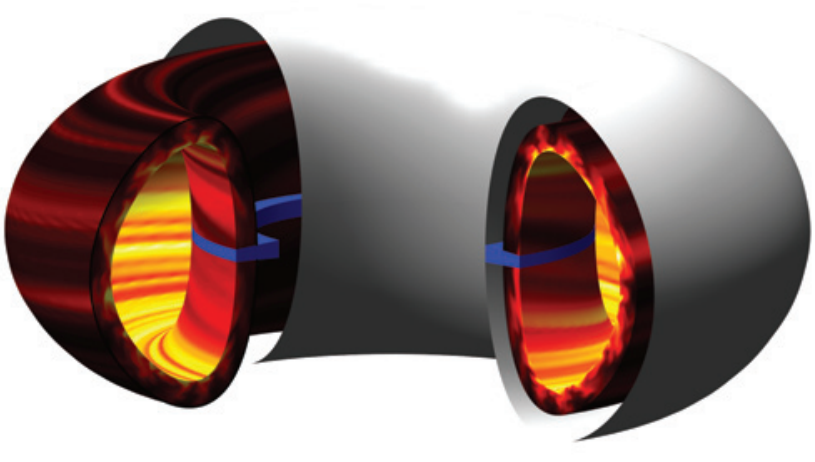

Figure 5 | Edge-region plasma simulation. Shown is a snapshot of plasma-pressure fluctuations from a fluid simulation of the outermost region of a tokamak plasma, where different shades of red indicate the amplitude of the perturbations, and the magnetic-field lines intersect the toroidal limiter (blue) inside the vessel. The turbulent structures, which are elongated in the direction of the magnetic field, lead to transport of density and heat from the core region towards the periphery of the device, while plasma is lost on the toroidal rail by flowing along the magnetic-field lines ${ }^{91}$

turbulent eddies, with practical implications on the self-regulation of turbulence and on the stabilizing effect of the conducting walls surrounding fusion plasmas. This spontaneous rotation is now attributed to turbulence, but the exact underlying mechanisms are still unclear, and predictions for ITER are still far from reliable.

As gyrokinetic codes become more and more realistic, their validation ${ }^{67-69}$ can be addressed with an increasing level of detail ${ }^{70,71}$ Results obtained within the local flux-tube approximation already give good results when applied to large tokamaks ${ }^{72}$, although limitations of the local approach are evident when applied to smaller devices ${ }^{73}$.

\section{Edge transport and plasma-wall interactions}

Some of the greatest uncertainties in ITER and future fusion reactors are related to the plasma dynamics in the SOL, the plasma-edge region $^{74-76}$, where the plasma is colder, contains multiple species and impurities (that is, ions other than deuterium or tritium ions), and is subject to interactions with the vessel-wall materials, and where the hydrogenic fuel and light impurities are incompletely ionized. The edge region plays a crucial role in the functioning of a fusion reactor; it influences the performance of the entire device, because it, for example, regulates the core-impurity level and has an impact on core turbulence and transport, particularly in small tokamaks.

Through local and non-local turbulence effects, plasma behaviour in this region governs key aspects of the overall confinement properties of the device. SOL phenomena regulate the impurity dynamics and the concentration of the $\alpha$-particles resulting from fusion reactions. Both can dilute the fusion fuel and stop the reactions. Moreover, SOL dynamics determines the heat load to the walls, a potential showstopper for fusion if material limits $\left(\sim 10 \mathrm{MW} \mathrm{m}^{-2}\right)$ are exceeded ${ }^{77,78}$.

Simulating SOL plasma dynamics is particularly challenging ${ }^{79}$. First, transport in the SOL is highly intermittent and, unlike in the tokamak core, is dominated by large fluctuations, the amplitudes of which are comparable to the equilibrium quantities. This does not allow a separation of scales between transport events and equilibrium profiles. Second, typical coordinate systems used for core-plasma simulations are singular in the SOL owing to the presence of the X-point, where the poloidal component of the magnetic field vanishes. Third, the plasma properties change significantly across the SOL (for example, the temperature in the SOL at JET varies from $\sim 1$ to $\sim 100 \mathrm{eV}$ (ref. 80 )), which makes it challenging to employ only one model for the description of the plasma-simultaneously taking into account very different collision frequencies is particularly difficult. Fourth, in contrast to the situation in the core region, strong gradients might develop also in the direction parallel to the magnetic field. Finally, the dynamics of neutral atoms and impurities as well as atomic and chemical processes play a key role ${ }^{75}$.

So far, wall components for future devices are designed largely on the basis of empirical extrapolations of plasma heat loads in present devices, supported by basic theoretical considerations ${ }^{74}$. In their simplest form, the codes solve a reduced set of fluid equations for the plasma density, electron and ion temperatures, and ion velocity in the direction parallel to the magnetic-field line. Transport perpendicular to the magnetic field is described by ad hoc diffusion-advection models and in the parallel direction by using collisional heat conductivity with corrections that mimic kinetic effects. The plasma description is coupled to Monte Carlo models for neutral particles to take into account vacuum pumping, fuelling and plasma-material interactions. The diffusion and convection coefficients are adjusted to fit the experimental data. These fits are then used to evaluate the SOL parameters, such as local density, temperature and diffusion coefficients, when the codes are run in predictive mode to study the dependence of the SOL profiles on the power entering the SOL from the core plasma, the pressure of the fusion fuel, the concentration of helium, the ratio between gas puffing and core fuelling, the pumping speed, and the magnetic geometry $^{74}$. As an example, the semi-empirical code $e^{81,82}$ used for the ITER divertor design ${ }^{83}$ is the result of an investment of over 100 professional years and consists of about 200,000 lines of source code $e^{84}$.

To predict the SOL properties in conditions very different from those in present devices, first-principles simulation codes are needed. Fully kinetic models are used to study SOL physics, as they allow a self-consistent description of the plasma, neutral atoms, and plasma-wall interfaces. These simulations have to bridge the spatial and temporal scales of electrostatic perturbations in plasmas, the Debye length and the plasma frequency $\left(\sim 10^{-5} \mathrm{~m}\right.$ and $10^{11} \mathrm{~s}^{-1}$, respectively), to the machine size and the typical turbulence frequencies $\left(\sim 1 \mathrm{~m}\right.$ and $10^{6} \mathrm{~s}^{-1}$, respectively). Therefore, they have an extremely high numerical cost and could so far be applied only to one-dimensional geometries ${ }^{85}$. Recently, the $3 \mathrm{D}$ simulation of SOL dynamics has been approached by extending global gyrokinetic models to cover the SOL domain ${ }^{86,87}$ and by also taking into account the effect of neutral particles using fluid codes. Starting from a set of fluid equations for collisional plasmas developed by Braginskii in $1965^{88}$, or by integrating the gyrokinetic equations in velocity space, a number of models more suited for computational treatment were deduced $^{89,90}$ and are now implemented in a number of codes ${ }^{91-93}$. Thanks to these simulations and to the relevant comparisons with increasingly accurate experimental information, significant progress has been made in our understanding of the physics underlying SOL turbulence. An example of a fluid simulation of the outermost plasma region of a tokamak device is shown in Fig. 5.

For scenarios with the simplest magnetic geometries, different turbulent regimes were identified ${ }^{94}$ together with the mechanisms that regulate the saturated levels of turbulence and transport ${ }^{95}$. These models can also provide an estimate of the SOL width, a key quantity determining the heat load on the plasma-facing components, and of its scaling with parameters such as density, temperature, magnetic field and major radius ${ }^{96}$. They also provide some insights into the origin of the spontaneous toroidal plasma rotation $^{97}$, the value of the electrostatic potential in the $\mathrm{SOL}^{98,99}$, and the dynamics of intermittent transport events ${ }^{100,101}$.

As turbulence in the SOL is dominated by modes that are strongly elongated in the direction parallel to the magnetic field, one can simplify the 3D equations and construct a 2D model that evolves the plasma dynamics in a plane perpendicular to the magnetic field, 


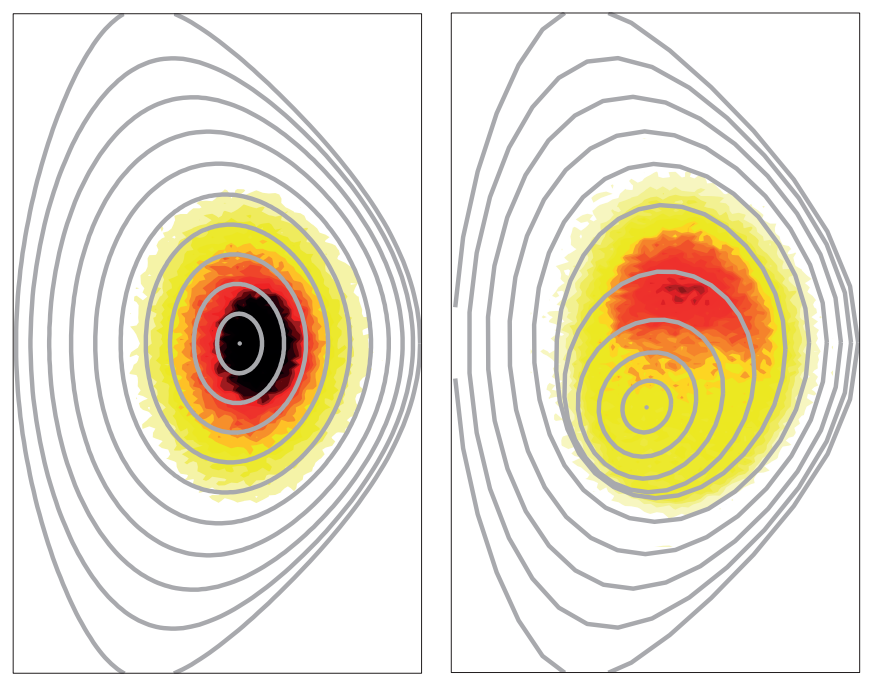

Figure 6 | Contours of the density distribution of neutral-beam-injected ions obtained from simulating their trajectories in the equilibrium magnetic field. a, The ions are injected into an axially symmetric plasma; the grey lines are cross-sections of the magnetic-flux surfaces in axially symmetric equilibrium. The colour map represents the local fast-ion density, which peaks close to the magnetic axis. $\mathbf{b}$, The ions are injected into an equilibrium with a 3D helical structure (perturbation) in the plasma core. The fast ions' density is highest where the magnetic-field lines are least compressed. The net result is that the energy density is peaked away from the magnetic axis, which has deleterious effects on plasma heating and current drive. Reproduced from ref. 40, IOP.

employing simplified models of the ignored parallel direction. Such approximated $2 \mathrm{D}$ models have been applied to study the radial propagation of 'blobs ${ }^{\text {' }}{ }^{2}$, plasma structures of enhanced density and temperature that can result in a significant transient load on the vessel walls. The code results are in good agreement with some of the experimentally observed turbulent properties ${ }^{103,104}$.

Most likely, fluid models will remain the workhorse of firstprinciples SOL simulations for a few more years. However, with the advancement of computational capabilities, we expect that gyrokinetic codes will eventually become the future tool of reference. This will also facilitate coupling of the SOL physics with the plasma dynamics inside the closed flux surface region, a crucial task, as a comprehensive description of the interplay between these two regions is necessary for the understanding of the overall plasma confinement.

\section{Fast ions}

Power-producing fusion reactors will be characterized by a fusion gain $Q$, the ratio of produced fusion power to input power to the plasma, which should be significantly larger than 10 for fusion reactors to be viable sources of energy. In DT plasmas, when the fusion gain approaches the range of interest for reactors $(Q>5)$, the energy released through the production of $3.5 \mathrm{MeV} \alpha$-particles provides the dominant contribution to the plasma heating-the plasma is said to be burning. Similarly to ions energized by NBI and ICRH, fusion-generated $\alpha$-particles constitute a strong suprathermal or 'fast' ion population, with energies much larger than the mean bulk plasma energy ${ }^{105}$. As the fast-ion slowing-down process by collisions occurs over macroscopic timescales ( $\sim 1 \mathrm{~s}$ in ITER), good confinement is essential to obtain a high fusion gain. In addition, as the fast ions carry large amounts of power (100 MW in the $Q=10,500 \mathrm{MW}$ power ITER reference scenario), only very minor $(<5 \%)$ losses can be tolerated.

Fast ions may be lost in the presence of imperfections in the confining field. These can be in the form of static magnetic perturbations due to magnetic ripple arising from the discrete number of toroidal field coils or to coils that deliberately break the toroidal symmetry to control certain MHD instabilities, or can be fluctuating electromagnetic fields arising from broadband turbulence or long-wavelength macroscopic MHD fluctuations.

Under certain conditions, fast ions themselves can drive MHD instabilities. Modes characterized by a very weak damping from the background plasma $(\gamma / \omega<1 \%$, where $\gamma$ and $\omega$ are the imaginary and real parts of the mode's frequency), such as Alfvén eigenmodes (AEs), specific solutions of the dispersion relation for Alfvén waves in tokamak geometry, are prone to instabilities driven by a relatively small population of fast ions that resonate with them ${ }^{106}$. In this situation, the nonlinear interaction between the electromagnetic disturbance and the fast-ion population can become important. Collisionless wave-particle resonant interactions ${ }^{107}$ explain the observed nonlinear cyclic behaviour of AEs and of the so-called 'fishbone instability', where the energetic-ion population causes a long-wavelength MHD mode to become unstable in the core. The same resonance causes cross-field transport of the ions, which in turn removes the instability drive and leads to a decay of the mode.

The standard MHD model captures most of the dynamics perpendicular to the equilibrium magnetic-field lines and allows one to calculate the main structure of the eigenmodes. To account for the resonant wave-particle interactions, a kinetic closure of the MHD equations is needed ${ }^{108,109}$. The resulting kinetic-MHD model describes more realistic dynamics parallel to the magnetic field for particles that are virtually collisionless over the timescales associated with the fluctuations of interest. Theoretical calculations of the damping of AEs performed using these models have been successfully compared with damping rates directly measured in the JET tokamak ${ }^{110}$. In addition to capturing the nonlinear physics of these unstable modes, codes that follow the guiding centres of fast ions in time-varying electromagnetic fields ${ }^{111}$ have also been used to calculate the stabilizing effect that fast ions can have on instabilities with the longest (nearly machine-scale) wavelength ${ }^{112}$.

Kinetic-MHD codes are often limited to following the guiding centres of the fast ions, and thus do not capture their interaction with fluctuating fields with wavelengths of the order of a gyroradius or less. In contrast, most gyrokinetic codes are typically limited to treating thermal populations (rather than fast ions) and electrostatic instabilities, and cannot treat long-wavelength, fully electromagnetic MHD fluctuations. However, recent advances in some gyrokinetic codes have enabled MHD instabilities in the Alfvén wave frequency range (of the order of $100-300 \mathrm{kHz}$ in ITER) to also be captured ${ }^{113-115}$.

A present challenge is to include fast ions in gyrokinetic codes to investigate how they interact with turbulence. Earlier simulations were simplified by treating fast ions as trace particles-particles the orbits of which are calculated but do not have any effect on other particles-with the objective of examining the effect of turbulence on $\alpha$-particle confinement ${ }^{116,117}$. Recent gyrokinetic simulations of fast ions in turbulent fields have removed the trace approximation, and have quantified the degree to which fast ions can damp ITG- and TEM-induced turbulence ${ }^{118}$. For some applications, fast varying fields necessitate a departure from gyroaveraging approximations and the deployment of full Lorentz ionorbit equations. A recent advance ${ }^{119}$ has been the implementation of curvilinear coordinate systems for fast and efficient full-orbit solvers in $3 \mathrm{D}$ fields, and efficient switching between guiding-centre and full-orbit trajectories ${ }^{120}$.

The stationary magnetic-field perturbations that break the toroidal symmetry of tokamak plasmas can also seriously degrade fast-ion confinement. Although the effects of magnetic ripple caused by the finite number of toroidal field coils have long been considered, a more recent concern is the deleterious effects of the coils used to control modes localized at the edge of the tokamak, 


\section{Box 1 | Reduced models for real-time control.}

The time required for simulations of fusion plasmas that employ relatively simple models and have been successfully used for decades by experimentalists in the preparation of plasma discharges ('runs') has decreased to the point that these simulations can now be used for real-time control. This enables physics-based predictive control, in which the physics information collected by the diagnostic measurements during a plasma discharge can be used as an input for simple simulation models, and the simulation results can then be immediately used in driving the discharge towards optimized performance and safe exploitation ${ }^{126-130}$. The main emphasis of these simulations is not on their accuracy, but rather on the shortest time to solution', because for real-time control applications, it is sufficient that the models and the simulations capture the main physics dependencies. The computational challenge is then to develop numerical schemes that work with a coarse grid and show monotonic convergence properties. The time required for these simulations should be $\sim 1 \mathrm{~ms}$, so that one can predict and drive the evolution of the plasma profiles, which evolve on the energy-confinement timescale (a few seconds in ITER).

Thanks to the improvement of numerical algorithms (and hardware), it is now possible to solve the nonlinear GradShafranov equation (equation (3)) and evaluate the plasma equilibrium magnetic field in less than $1 \mathrm{~ms}$, taking into account the currents flowing in the plasma, the coils and the wall ${ }^{13}$. The electron-cyclotron beam trajectory and its interaction with the plasma can also be calculated on a similar timescale ${ }^{131}$. For other problems, such as solving the transport equations that describe the evolution of the pressure and current profiles, new models need to be developed specifically for real-time control ${ }^{129,132}$.

The critical significance of the simulation turnover time dictates the choice of the platform on which the analysis of the diagnostic data and the simulations are performed. Graphical processor units (GPUs) are being used to speed up dedicated applications, such as the treatment of data from a complex diagnostic. In these cases, the overhead time related to setting up the parallel processes, moving data and collecting the results, needs to be carefully engineered.

An example of a code developed for real-time control is RAPTOR $^{129}$, now applied to several tokamak experiments, which is able to simulate the evolution of the radial profiles of electron temperature and current density-two crucial quantities for the stability and the performance of a plasma discharge. The RAPTOR code is executed within the real-time control algorithm in parallel with the tokamak discharge. Whereas the plasma profile is maintained according to the discharge specifications, the growth

and of fields associated with internal perturbations ${ }^{121}$, which often occur in plasma scenarios designed to avoid sawtooth oscillations. These are now well modelled in simulations of the distribution of the NBI ion population in the 3D equilibrium magnetic field associated with these perturbations ${ }^{40}$; see Fig. 6 for an illustration. The predicted reduction in the confinement of fast ions due to the $3 \mathrm{D}$ fields has been compared favourably with experimental measurements. In simulations of this type, the $3 \mathrm{D}$ fields in the orbitequation solver must be represented very accurately, because the fast ions must be followed until they are thermalized, that is, over a macroscopic timescale, and numerical errors would propagate, leading to unphysical results ${ }^{119}$.

A significant remaining challenge for modelling energetic-ion confinement in static fields relates to identifying the most accurate of localized modes that severely limit the performance of a discharge is kept under control by driving current with localized electron-cyclotron wave injection.

The figure shows an example of the application to the combined control of current density and temperature profiles in the TCV tokamak. The discharge trajectory-that is, the time evolution of the external actuators and plasma parameters-is optimized with respect to general goals such as maximizing fusion performance and radiation levels or minimizing forces in case of disruption ${ }^{133}$.
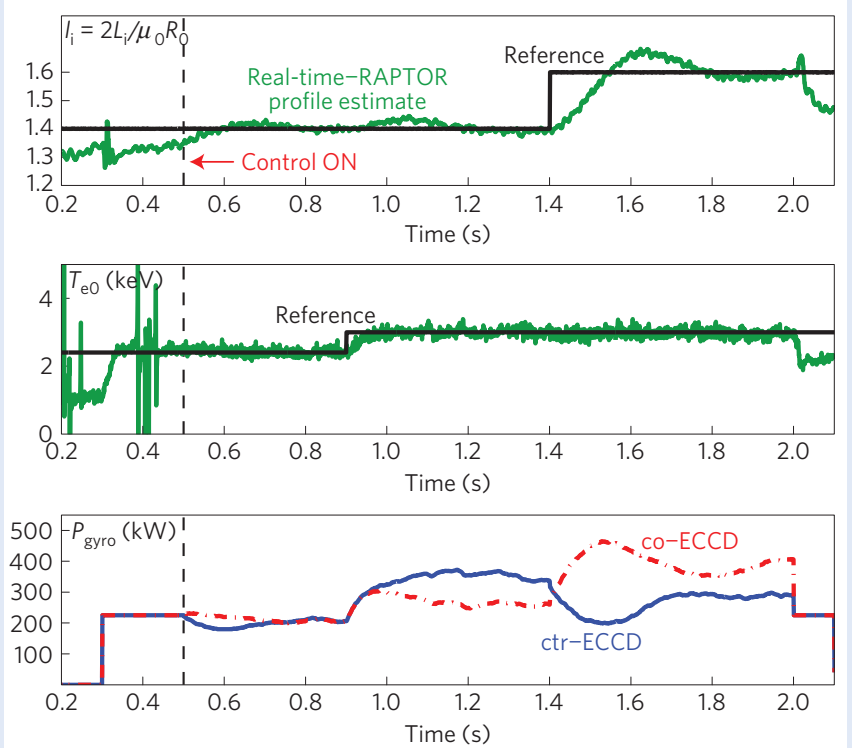

Real-time simulations help controlling a plasma discharge.

Simultaneous control of the current profile (top) and the central electron temperature $T_{\mathrm{e} 0}$ (middle, measured in this case from X-ray emission) using two ECCD sources in co- and counter-current drive in the TCV tokamak. Active control is switched on at $t=0.5 \mathrm{~s}$ in the discharge. Current profile peaking is quantified by the non-dimensional plasma internal inductance $I_{i}=2 L_{i} / \mu_{0} R_{0}$, calculated from the dimensional value of the inductance $L_{i}$, with $R_{0}$ being the tokamak major radius and $\mu_{0}$ the permeability of vacuum. When the reference temperature $T_{\mathrm{e} 0}$ is increased, the total power increases to match the demand with equal coand counter-current contributions, thus avoiding changing the current density profile. On the other hand, when the reference inductance $l_{i}$ is increased, more co-current drive is added, at constant total power (bottom), thus peaking the current density profile while avoiding a change in $T_{\mathrm{e} 0}$ (ref. 128). Figure reproduced with permission from ref. 128.

model for representing the plasma response to fields that break axial symmetry $^{122,123}$.

\section{Final remarks}

The field of numerical modelling of fusion plasmas is still very open, yet it is fair to say that it stands out as a success story. A number of issues can be considered essentially solved. Examples include macroscopic equilibrium and stability in tokamaks in the MHD approximation, collisional transport, and a number of plasma heating and current-drive schemes. However, the intrinsic complexity, multi-scale and multi-physics nature of plasmas still prevents a comprehensive description of all the phenomena of relevance for attaining and controlling the burning-plasma regime. The numerical simulations that are available today are necessarily 
based on a hierarchy of models, each addressing specific aspects of plasma dynamics. The present trend is to combine the codes that treat the separate (though interacting) physical phenomena occurring in a plasma to achieve a comprehensive understanding of the behaviour of a fusion-plasma discharge. A slighty more futuristic approach is, on the other hand, to construct a single code that would ultimately include all essential physical phenomena from the edge to the core plasma. Striking the balance between the two methodologies is a major challenge in itself, and has motivated a number of nationally and internationally coordinated initiatives, which accelerate progress by applying an interdisciplinary approach that involves applied mathematicians, analytical and computational physicists, computer scientists, and experts of real-time control (see Box 1), complex systems and big-data science.

\section{Received 20 October 2015; accepted 24 March 2016;} published online 3 May 2016

\section{References}

1. Ongena, J., Koch, R., Wolf, R. \& Zohm., H. Magnetic-confinement fusion. Nature Phys. 12, 398-410 (2016).

2. Shimada, M. et al. Progress in the ITER physics basis-Chapter 1: Overview and summary. Nucl. Fusion 47, S1-S17 (2007).

3. Freidberg, J. P. Ideal MHD (Plenum Press, 1987).

4. Victoria, M. et al. Modelling irradiation effects in fusion materials. Fusion Eng. Des. 82, 2413-2421 (2007).

5. Knaster, J., Moeslang, A. \& Muroga, T. Materials research for fusion. Nature Phys. 12, 424-434 (2016).

6. Dudarev, S. L. Density functional theory models for radiation damage. Rev. Mater. Res. 43, 35-61 (2013).

7. Hender, T. C. et al. Progress in the ITER physics basis-Chapter 3: MHD stability, operational limits and disruptions. Nucl. Fusion 47, S128-S202 (2007).

8. Freidberg, J. P. Plasma Physics and Fusion Energy (Cambridge Univ. Press, 2007).

9. Shafranov, V. D. On magnetohydrodynamical equilibrium configurations. Sov. Phys. JETP 6, 545-554 (1958).

10. Lüst, R. \& Schlüter, A. Axisymmetrische magnetohydrodynamische Gleichgewichtskonfigurationen. Z. Naturforsch. 12a, 850-854 (1957).

11. Grad, H. \& Rubin, H. Hydromagnetic equilibria and force-free fields. in Proc. 2nd Int. Conf. Peaceful Uses of Atomic Energy Vol. 31, 190-197 (United Nations, 1958).

12. Ferron, J. R. et al. Real time equilibrium reconstruction for tokamak discharge control. Nucl. Fusion 38, 1055-1066 (1998)

13. Moret, J.-M. et al. Tokamak equilibrium reconstruction code LIUQE and its real time implementation. Fusion Eng. Des. 91, 1-15 (2015).

14. Treutterer, W. et al. Integrated operation of diagnostic and control systems. Fusion Eng. Des. 86, 465-470 (2011).

15. Reich, M. et al. ASDEX Upgrade team, Real time beam tracing for control of the deposition location of electron cyclotron waves. Fusion Eng. Des. 100, 73-80 (2015).

16. Lütjens, H., Bondeson, A. \& Sauter, O. The CHEASE code for toroidal MHD equilibria. Comput. Phys. Commun. 97, 219-260 (1996).

17. Troyon, F., Gruber, R., Saurenmann, H., Semenzato, S. \& Succi, S. MHD limits to plasma confinement. Plasma Phys. Control. Fusion 26, 209-215 (1984).

18. Turnbull, A. D., Roy, A., Sauter, O. \& Troyon, F. Current and beta-limitations for the TCV tokamak. Nucl. Fusion 28, 1379-1383 (1988).

19. Hofmann, F., Sauter, O., Reimerdes, H., Furno, I. \& Pochelon, A. Experimental and theoretical stability limits of highly elongated tokamak plasmas. Phys. Rev. Lett. 81, 2918-2921 (1998)

20. Brunetti, D., Graves, J. P., Cooper, W. A., Terranova, D. \& Wahlberg, C. Fast growing instabilities and non-linear saturated states in hybrid tokamak and RFP plasmas. Nucl. Fusion 54, 064017 (2014).

21. Strait, E. J. et al. Wall stabilization of high Beta tokamak discharges in DIII-D. Phys. Rev. Lett. 74, 2483-2486 (1995).

22. Grieger, G. et al. Physics optimization of stellarators. Phys. Fluids B 4, 2081-2091 (1992)

23. Nuhrenberg, J. \& Zille, R. Quasi-helically symmetric toroidal stellarators. Phys. Lett. A 129, 113-117 (1988).

24. Nuhrenberg, J., Lotz, W. \& Gori, S. Quasi-Axisymmetric Tokamaks. in Theory of Fusion Plasmas: Proc. Joint Varenna-Lausanne Int. Workshop (eds Sindoni, E., Troyon, F. \& Vaclavik, J.) 3-12 (International School of Plasma Physics 'Piero Caldirola' Series, Italian Physical Society, 1994).
25. Mynick, H. E. Transport optimization in stellarators. Phys. Plasmas 13, 058102 (2006).

26. Boozer, A. H. Guiding center drift equations. Phys. Fluids 23, 904-908 (1980).

27. Nuhrenberg, J. Development of quasi-isodynamic stellarators. Plasma Phys. Control. Fusion 52, 124003 (2010).

28. Wanner, M. \& the W7X Team. Design goals and status of W7X project. Plasma Phys. Control. Fusion 42, 1179-1186 (2000).

29. Kadomstev, B. B. \& Pogutse, O. P. in Reviews of Plasma Physics Vol. 5 (ed. Leontivitch, M. A.) 249-400 (Consultants Bureau, 1970).

30. Cooper, W. A., Graves, J. P. \& Sauter, O. JET snake magnetohydrodynamic equilibria. Nucl. Fusion 51, 072002 (2011).

31. Cooper, W. A. et al. Magnetohydrodynamic equilibrium and the stability of tokamaks and reversed-field pinch systems with 3D helical cores. Plasma Control. Fusion 53, 074008 (2011).

32. Mazon, D., Fenzi, C. \& Sabot, R. As hot as it gets. Nature Phys. 12, 14-17 (2016)

33. Stix, T. H. \& Nierenberg, W. The Theory of Plasma Waves (Literacy Licensing LLC, 2012).

34. Kazakov, Ye. O., van Eester, D., Dumont, R. \& Ongena, J. On resonant ICRF absorption in three-ion component plasmas: a new promising tool for fast ion generation. J. Nucl. Fusion 55, 032001 (2015).

35. Jaeger, E. F. et al. Self-consistent full-wave and Fokker-Planck calculations for ion cyclotron heating in non-Maxwellian plasmas. Phys. Plasmas 13, 056101 (2006).

36. Dumont, R. J. \& Zarzoso, D. Heating and current drive by ion cyclotron waves in the activated phase of ITER. Nucl. Fusion 53, 013002 (2013).

37. Jucker, M. et al. Integrated modeling for ion cyclotron resonant heating in toroidal systems. Comput. Phys. Commun. 182, 912-925 (2011).

38. Mayoral, M.-L. et al. On the challenge of plasma heating with the JET metallic wall. Nucl. Fusion 54, 033002 (2014).

39. Faustin, J. et al. Applications of the SCENIC code package to the minority ion-cyclotron heating in Wendelstein 7-X plasmas. 1689, 060003 (AIP, 2015).

40. Pfefferlé, D. et al. NBI fast ion confinement in the helical core of MAST hybrid-like plasmas. Nucl. Fusion 54, 064020 (2014).

41. Prater, R. et al. Summary of Papers on ECH and ECCD. Nucl. Fusion 48, 035006 (2008).

42. Sauter, O. et al. Steady-state fully non-inductive current driven by electron cyclotron waves in a magnetically confined Plasma. Phys. Rev. Lett. 84, 3322-3325 (2000).

43. Poli, E. et al. On recent results in the modelling of neoclassical-tearing-mode stabilization via electron cyclotron current drive and their impact on the design of the upper EC launcher for ITER. Nucl. Fusion 55, 013023 (2015).

44. Garbet, X., Idomura, Y., Villard, L. \& Watanabe, T. H. Gyrokinetic simulations of turbulent transport. Nucl. Fusion 50, 043002 (2010).

45. Horton, W. Drift waves and transport. Rev. Mod. Phys. 71, 735-778 (1999).

46. Brizard, A. \& Hahm, T. S. Foundations of nonlinear gyrokinetic theory. Rev. Mod. Phys. 79, 421-468 (2007).

47. Frieman, E. A. \& Chen, L. Non-linear gyrokinetic equations for low-frequency electromagnetic waves in general plasma equilibria. Phys. Fluids 25, 502-508 (1982).

48. Abel, I. G. et al. Multiscale gyrokinetics for rotating tokamak plasmas: fluctuations, transport and energy flows. Rep. Prog. Phys. 75, 116201 (2013).

49. Sugama, H. Gyrokinetic field theory. Phys. Plasmas 7, 466-480 (2000).

50. Krommes, J. A. The gyrokinetic description of microturbulence in magnetized plasmas. Annu. Rev. Fluid Mech. 44, 175-201 (2012).

51. Lee, W. W. Gyrokinetic particle simulation model. J. Comput. Phys. 72, 243-269 (1987).

52. Lin, Z., Hahm, T. S., Lee, W. W., Tang, W. \& White, R. Turbulent transport reduction by zonal flows: massively parallel simulations. Science 281, 1835-1837 (1998).

53. Itoh, K. et al. Physics of zonal flows. Phys. Plasmas 13, 055502 (2006).

54. Jolliet, S. et al. A global collisionless PIC code in magnetic coordinates. Comput. Phys. Commun. 177, 409-425 (2007).

55. Kotschenreuther, M., Rewoldt, G. \& Tang, W. M. Comparison of initial value and eigenvalue codes for kinetic toroidal plasma instabilities. Comput. Phys. Commun. 88, 128-140 (1995).

56. Jenko, F., Dorland, W., Kotschenreuther, M. \& Rogers, B. N. Electron temperature gradient driven turbulence. Phys. Plasmas 7, 1904-1910 (2000).

57. Candy, J. \& Waltz, R. E. An Eulerian gyrokinetic-Maxwell solver. J. Comput. Phys. 186, 545-581 (2003).

58. Grandgirard, V. et al. A drift-kinetic semi-Lagrangian $4 \mathrm{D}$ code for ion turbulence simulation. J. Comput. Phys. 217, 395-423 (2006).

59. Beer, M. A., Cowley, S. C. \& Hammett, G. W. Field-aligned coordinates for nonlinear simulations of tokamak turbulence. Phys. Plasmas 2, 2687-2700 (1995). 
60. Mishchenko, A. et al. Global linear gyrokinetic particle-in-cell simulations including electromagnetic effects in shaped plasmas. Nucl. Fusion 55, 053006 (2015).

61. Startsev, E. A. \& Lee, W. W. Finite beta simulation of microinstabilities. Phys Plasmas 21, 022505 (2014).

62. Maeyama, S. et al. Cross-scale interactions between Electron and ion scale turbulence in a Tokamak plasma. Phys. Rev. Lett. 114, 255002 (2015).

63. Goerler, T. \& Jenko, F. Scale separation between electron and ion thermal transport. Phys. Rev. Lett. 100, 185002 (2008).

64. Lin, Z., Ethier, S., Hahm, T. S. \& Tang, W. M. Size scaling of turbulent transport in magnetically confined plasmas. Phys. Rev. Lett. 88, 195004 (2002)

65. McMillan, B. F., Lapillonne, X., Brunner, S. \& Villard, L. System size effects on Gyrokinetic turbulence. Phys. Rev. Lett. 105, 155001 (2010)

66. Rice, J. E. et al. Observations of anomalous momentum transport in Alcator C-Mod plasmas with no momentum input. Nucl. Fusion 44, 379-386 (2004)

67. Oberkampf, W. L. \& Roy, C. J. Verification and Validation in Scientific Computing (Cambridge Univ. Press, 2010).

68. Greenwald, M. Verification and validation for magnetic fusion. Phys. Plasmas 17, 058101 (2010).

69. Ricci, P. et al. Methodology for turbulence code validation: quantification of simulation-experiment agreement and application to the TORPEX experiment. Phys. Plasmas 18, 032109 (2011)

70. Holland, C. et al. Validation studies of gyrofluid and gyrokinetic predictions of transport and turbulence stiffness using the DIII-D tokamak. Nucl. Fusion 53, 083027 (2013).

71. Howard, N. T., Holland, C., White, A. E., Greenwald, M. \& Candy, J. Fidelity of reduced and realistic electron mass ratio multi-scale gyrokinetic simulations of tokamak discharges. Plasma Phys. Control. Fusion 57, 065009 (2015).

72. Citrin, J. et al. Ion temperature profile stiffness: non-linear gyrokinetic simulations and comparison with experiment. Nucl. Fusion 54, 023008 (2014)

73. Merlo, G. et al. Investigating profile stiffness and critical gradients in shaped TCV discharges using local gyrokinetic simulations of turbulent transport. Plasma Phys. Control. Fusion 57, 054010 (2015).

74. Loarte, A. et al. Progress in the ITER physics basis-Chapter 4: Power and particle control. Nucl. Fusion 47, S203-S263 (2007).

75. Stangeby, P. C. The Plasma Boundary of Magnetic Fusion Devices (Institute of Physics Publishing, 2000).

76. ITER Physics Expert Group on Divertor, ITER Physics Expert Group on Divertor Modelling and Database and ITER Physics Basis Editors Chapter 4 Power and particle control. Nucl. Fusion 39, 2391-2469 (1999).

77. Zohm, H. et al. On the physics guidelines for a tokamak demo. Nucl. Fusion 53, 073019 (2013)

78. Eich, T. et al. Inter-ELM power decay length for JET and ASDEX Upgrade: measurement and comparison with heuristic drift-based model. Phys. Rev. Lett. 107, 215001 (2011).

79. Ricci, P. Simulation of the scrape-off layer region of tokamak devices. J. Plasma Phys. 81, 435810202 (2015).

80. Erents, S. K. \& Stangeby, P. C. Heat transport in the JET scrape-off layer. Nucl. Fusion 38, 1637-1650 (1998).

81. Reiter, D. et al. Helium removal from tokamaks. Plasma Phys. Control. Fusion 33, 1579-1600 (1991).

82. Schneider, R. et al. B2-eirene simulation of ASDEX and Asdex-upgrade scrape-off layer plasmas. J. Nucl. Mater. 196-198, 810-815 (1992).

83. Pitts, R. A. et al. Status and physics basis of the ITER divertor. Phys. Scr. T138 014001 (2009)

84. Kukushkin, A. S., Pacher, H. D., Kotov, V., Pacher, G. W. \& Reiter, D. Finalizing the ITER divertor design: the key role of SOLPS modeling. Fusion Eng. Des. 86, 2865-2873 (2011).

85. Tskhakaya, D. On recent massively parallelized PIC simulations of the SOL Contrib. Plasma Phys. 52, 490-499 (2012).

86. Chang, C. S. et al. Whole-volume integrated gyrokinetic simulation of plasma turbulence in realistic diverted-tokamak geometry. J. Phys. Conf. Ser. 180, 012057 (2009).

87. Seo, J. et al. Intrinsic momentum generation by a combined neoclassical and turbulence mechanism in diverted DIII-D plasma edge. Phys. Plasmas 21, 092501 (2014)

88. Braginskii, S. I. in Reviews of Plasma Physics Vol. 1 (ed. Leontovich, M. A.) 205-311 (Consultants Bureau, 1965).

89. Zeiler, A., Drake, J. F. \& Rogers, B. Nonlinear reduced Braginskii equations with ion thermal dynamics in toroidal plasma. Phys. Plasmas 4, 2134-2138 (1997).

90. Ribeiro, T. T. \& Scott, B. Tokamak turbulence computations on closed and open magnetic flux surfaces. Plasma Phys. Control. Fusion 47, 1657-1679 (2005).
91. Ricci, P. et al. Simulation of plasma turbulence in scrape-off layer conditions: the GBS code, simulation results and code validation. Plasma Phys. Control. Fusion 54, 124047 (2012).

92. Dudson, B. D., Umansky, M. V., Xu, X. Q., Snyder, P. B. \& Wilson, H. R. BOUT++: a framework for parallel plasma fluid simulations. Comput. Phys. Commun. 180, 1467-1480 (2009).

93. Tamain, P. et al. Tokam-3d: A 3d fluid code for transport and turbulence in the edge plasma of tokamaks. J. Comput. Phys. 229, 361-378 (2010).

94. Mosetto, A., Halpern, F., Jolliet, S., Loizu, J. \& Ricci, P. Turbulent regimes in the tokamak scrape-off layer. Phys. Plasmas 20, 092308 (2013).

95. Ricci, P. \& Rogers, B. Plasma turbulence in the scrape-off layer of tokamak devices. Phys. Plasmas 20, 010702 (2013).

96. Halpern, F. D., Ricci, P., Jolliet, S., Loizu, J. \& Mosetto, A. Theory of the scrape-off layer width in inner-wall limited tokamak plasmas. Nucl. Fusion 54, 043003 (2014).

97. Loizu, J., Ricci, P., Halpern, F. D., Jolliet, S. \& Mosetto, A. Intrinsic toroidal rotation in the scrape-off layer of tokamaks. Phys. Plasmas 21, 062309 (2014)

98. Loizu, J., Ricci, P., Halpern, F. D., Jolliet, S. \& Mosetto, A. On the electrostatic potential in the scrape-off layer of magnetic confinement devices. Plasma Phys. Control. Fusion 55, 124019 (2013).

99. Halpern, F. D. et al. Contributors, JET-EFDA Theory-based scaling of the sol width in circular limited tokamak plasmas. Nucl. Fusion 53, 122001 (2013).

100. Xu, X. Q., Dudson, B., Snyder, P. B., Umansky, M. V. \& Wilson, H. Nonlinear simulations of peeling-ballooning modes with anomalous electron viscosity and their role in edge localized mode crashes. Phys. Rev. Lett. 105, 175005 (2010)

101. Walkden, N. R., Hudson, B. D., Easy, L., Fishpool, G. \& Omotani, J. T. Numerical investigation of isolated filament motion in a realistic tokamak geometry. Nucl. Fusion 55, 113022 (2015).

102. D’Ippolito, D. A., Myra, J. R. \& Zweben, S. J. 2011 Convective transport by intermittent blob-filaments: comparison of theory and experiment. Phys. Plasmas 18, 060501 (2011)

103. Russell, D. A. et al., The NSTX Team. Comparison of scrape-off layer turbulence simulations with experiments using a synthetic gas puff imaging diagnostic. Phys. Plasmas 18, 022306 (2011).

104. Garcia, O. E. et al. Interchange turbulence in the TCV scrape-off layer. Plasma Phys. Control. Fusion 48, L1-L10 (2006).

105. Fasoli, A. et al. Progress in the ITER Physics Basis Chapter 5: Physics of energetic ions. Nucl. Fusion 47, S264-S284 (2007).

106. Chen, L. \& Zonca, F. Physics of Alfvén waves and energetic particles in burning plasmas. Rev. Mod. Phys. 88, 015008 (2016)

107. Chen, L., White, R. B. \& Rosenbluth, M. N. Excitation of internal kink modes by trapped energetic beam ions. Phys. Rev. Lett. 52, 1122-1125 (1984).

108. Park, W. et al. Three-dimensional hybrid gyrokinetic-magnetohydrodynamics simulation. Phys. Fluids B 4, 2033-2037 (1992).

109. Cheng, C. Z. A kinetic-magnetohydrodynamic model for low-frequency phenomena. J. Geophys. Res. 96, 21159-21171 (1991).

110. Fasoli, A. et al. Direct measurement of the damping of toroidicity-induced Alfvén eigenmodes. Phys. Rev. Lett. 75, 645-648 (1995).

111. Pinches, S. D. et al. The HAGIS self-consistent nonlinear wave-particle interaction model. Comput. Phys. Commun. 111, 133-149 (1998).

112. Graves, J. P. et al. Control of magnetohydrodynamic stability by phase space engineering of energetic ions in tokamak plasmas. Nature Commun. 3, 624 (2012)

113. Bass, E. M. \& Waltz, R. E. Gyrokinetic simulation of global and local Alfvén eigenmodes driven by energetic particles in a DIII-D discharge. Phys. Plasmas 20, 012508 (2013).

114. Deng, W. et al. Gyrokinetic particle simulations of reversed shear Alfvén eigenmodes excited by antenna and fast ions. Phys. Plasmas $\mathbf{1 7}$ 112504 (2010)

115. Lauber, P. Local and global kinetic stability analysis of Alfvén eigenmodes in the 15 MA ITER scenario. Plasma Phys. Control. Fusion 57, 054011 (2015).

116. Zhang, W., Lin, Z. \& Chen, L. Transport of Energetic Particles by Microturbulence in Magnetized Plasmas. Phys. Rev. Lett. 101, 095001 (2008).

117. Albergante, M., Fasoli, A., Graves, J. P., Brunner, S. \& Cooper, W. A. Assessment of turbulent beam ion redistribution in tokamaks through velocity space dependent gyrokinetic analyses. Nucl. Fusion 52, 094016 (2012).

118. Citrin, J. et al. Nonlinear stabilization of tokamak microturbulence by fast ions. Phys. Rev. Lett. 111, 155001 (2013)

119. Pfefferlé, D., Cooper, W. A., Graves, J. P. \& Misev, C. Venus-LEVIS and its spline-Fourier interpolation of $3 \mathrm{D}$ toroidal magnetic field representation for guiding-centre and full-orbit simulations of charged particles. Comput. Phys. Commun. 185, 3127-3140 (2014).

120. Pfefferlé, D., Graves, J. P. \& Cooper, W. A. Hybrid guiding-centre/full-orbit simulations in non-axisymmetric magnetic geometry exploiting general criterion for guiding-centre accuracy. Plasma Phys. Control. Fusion 57, 054017 (2015). 
121. Chapman, I. T. et al., The MAST Team. Saturated ideal modes in advanced tokamak regimes in MAST. Nucl. Fusion 50, 045007 (2010).

122. Turnbull, A. D. et al. Comparisons of linear and nonlinear plasma response models for non-axisymmetric perturbations. Phys. Plasmas 20, 056114 (2013).

123. Pfefferlé, D., Misev, C., Cooper, W. A. \& Graves, J. P. Impact of RMP magnetic field simulation models on fast ion losses. Nucl. Fusion 55, 012001 (2015).

124. ITER Physics Expert Group on Disruptions, Plasma Control, and MHD ITER Physics Basis Editors. Chapter 3: MHD stability, operational limits and disruptions. Nucl. Fusion 39, 2251-2389 (1999).

125. Villard, L. et al. Global gyrokinetic ion temperature gradient turbulence simulations of ITER. Plasma Phys. Control. Fusion 55, 074017 (2013).

126. Ou, Y. et al. Towards model-based current profile control at DIII-D. Fusion Eng. Des. 82, 1153-1160 (2007).

127. Witrant, E. et al. A control-oriented model of the current profile in tokamak plasma. Plasma Phys. Control. Fusion 49, 1075-1105 (2007).

128. Felici, F. Real-Time Control of Tokamak Plasmas: from Control of Physics to Physics-Based Control PhD thesis, EPFL No. 5203 (2011); http://dx.doi.org/10.5075/epfl-thesis-5203

129. Felici, F. et al. The TCV Team. Real-time physics-model-based simulation of the current density profile in tokamak plasmas. Nucl. Fusion $\mathbf{5 1}$, 083052 (2011)
130. Humphreys, D. et al. Novel aspects of plasma control in ITER. Phys. Plasmas 22, 021806 (2015).

131. Reich, M. et al. Real-time TORBEAM. Fusion Eng. Des. 100, 73-80 (2015)

132. Artaud, J. et al. The CRONOS suite of codes for integrated tokamak modeling. Nucl. Fusion 50, 043001 (2010)

133. Felici, F. \& Sauter, O. Nonlinear model-based optimization of actuator trajectories for tokamak plasma profile control. Plasma Phys. Control. Fusion 54, 025002 (2012).

\section{Acknowledgements}

The authors wish to thank T.-M. Tran for his long-lasting support on HPC matters, and J. Faustin, D. Pfefferlé and F. Rive for help with the figure preparation.

\section{Additional information}

Reprints and permissions information is available online at www.nature.com/reprints. Correspondence should be addressed to A.F.

\section{Competing financial interests}

The authors declare no competing financial interests. 